Unfallchirurg 2018 · 121:884-892

https://doi.org/10.1007/s00113-018-0549-9

(c) Der/die Autor(en) 2018

Redaktion

C. Krettek, Hannover

CrossMark

\section{Christian Krettek}

Medizinische Hochschule Hannover (MHH), Hannover, Deutschland

\title{
MagicTube: Neue Möglichkeiten zum komplett internen Knochensegmenttransport und optionaler Verlängerung
}

\section{Neues Zusatzmodul für motorisierte Verlängerungsnägel zur Behandlung großer Knochendefekte}

\begin{abstract}
Knochensegmentdefekte und Extremitätenverkürzungen stellen schwerwiegende Probleme in der muskuloskeletalen Chirurgie dar. Während für Extremitätenverkürzungen in den letzten Jahren neue und zunehmend bessere motorisierte Verlängerungsimplantate zur komplett internen Anwendung entwickelt wurden, standen für Knochendefekte keine entsprechenden, breit anwendbaren Verfahren zur Verfügung. Zur Lösung dieses Problems wurde ein Implantatmodul entwickelt, das mithilfe motorisierter Verlängerungsimplantate einen komplett internen Knochensegmenttransport (KST) und, sofern erforderlich, optional auch eine zusätzliche Knochenverlängerung ohne weiteren Eingriff erlaubt.
\end{abstract}

\section{Grundlagen}

Segmentale Knochendefekte können aus den unterschiedlichsten Gründen entstehen, z. B. als Folge von offenen Frakturen und Hochrasanztraumen mit Knochenverlust, als Folge von chirurgischem knöchernem Débridement bei devitalisiertem oder infiziertem Knochen sowie bei Tumoren und anderen pathologischen Veränderungen. $\mathrm{Zu}$ den Behandlungsmethoden gehören $u$. a. die Transplantation von autologem Kno- chen, die Verwendung von Knochenersatzmaterialien, die Verkürzung, die Verkürzung mit sekundärer Verlängerung, der Einsatz von Überbrückungsimplantaten und der KST. Der KST kann mit einem komplett externen Fixierungsund Transportsystem [3, 12] oder als externes Transportsystem entlang eines inneren Stabilisierungssystems wie z.B. einem intramedullären Nagel [14] oder einer Platte $[8,11]$ durchgeführt werden. Die Entwicklung der externen Fixation war eine großartige Ergänzung zur Behandlung von Knochendefekten im Bewegungsapparat. Externe Fixateure können aber mit einer Vielzahl von Nachteilen, Problemen oder Komplikationen verbunden sein [12]. Dazu gehören Schmerzen, „Pin-tract“-Infektion, Gelenkeinsteifung und Kontrakturen, Störung des Gangbilds, eingeschränkter Tragekomfort und eine Vielzahl von ästhetischen und psychischen Problemen. Insbesondere bestehen diese Probleme am Oberschenkel. Erfolgt der KST über ein externes Transportsystem entlang von inneren Implantaten wie einem Nagel oder einer Platte, wird die Zeit, die im externen Fixateur benötigt wird, um etwa $30 \%$ reduziert, verglichen mit einem alleinigen externen Transport und Fixationssystem.

In jüngster Zeit wurden motorisierte Nägel für die Verlängerung von Gliedmaßen $[2,6,7]$ beschrieben. Der Motor des Nagels kann mithilfe mechanischer Energie durch Gang oder mithilfe elektrischer Energie entweder aus einer Stromquelle oder magnetisch mit einer speziellen Induktionsvorrichtung betrieben werden. Ein eigens angefertigter motorisierter Nagel für den KST wurde in der Vergangenheit in einem Fall beschrieben [1].

Mithilfe des MagicTube kann ohne zusätzliche Operation vom KST in die Verlängerung übergegangen werden

In diesem Beitrag wird die Behandlung von Knochendefekten mit dem neu entwickelten einfachen universellen Implantatmodul Cylinder-Kombi-Tubus für segmentalen Transport (CKTST) oder kurz MagicTube (Modaleres Assistenz Gerät für Intersegmentale Corticalisdefekte mittels Tubus Erweiterung) [5], beschrieben (Hersteller: K-Implant GmbH, 30826 Garbsen). Das Modul kann an jeden motorisierten Verlängerungsnagel angepasst und aufgesteckt werden ( $\bullet$ Abb. 1 und 2). Dieses Modul nutzt die Antriebskraft und Steuerung (vorwärts-, Stopp-, rückwärts- und Geschwindigkeitsregelung) eines motorisierten Verlängerungsnagels für den KST und ermöglicht eine optionale zusätzli- 


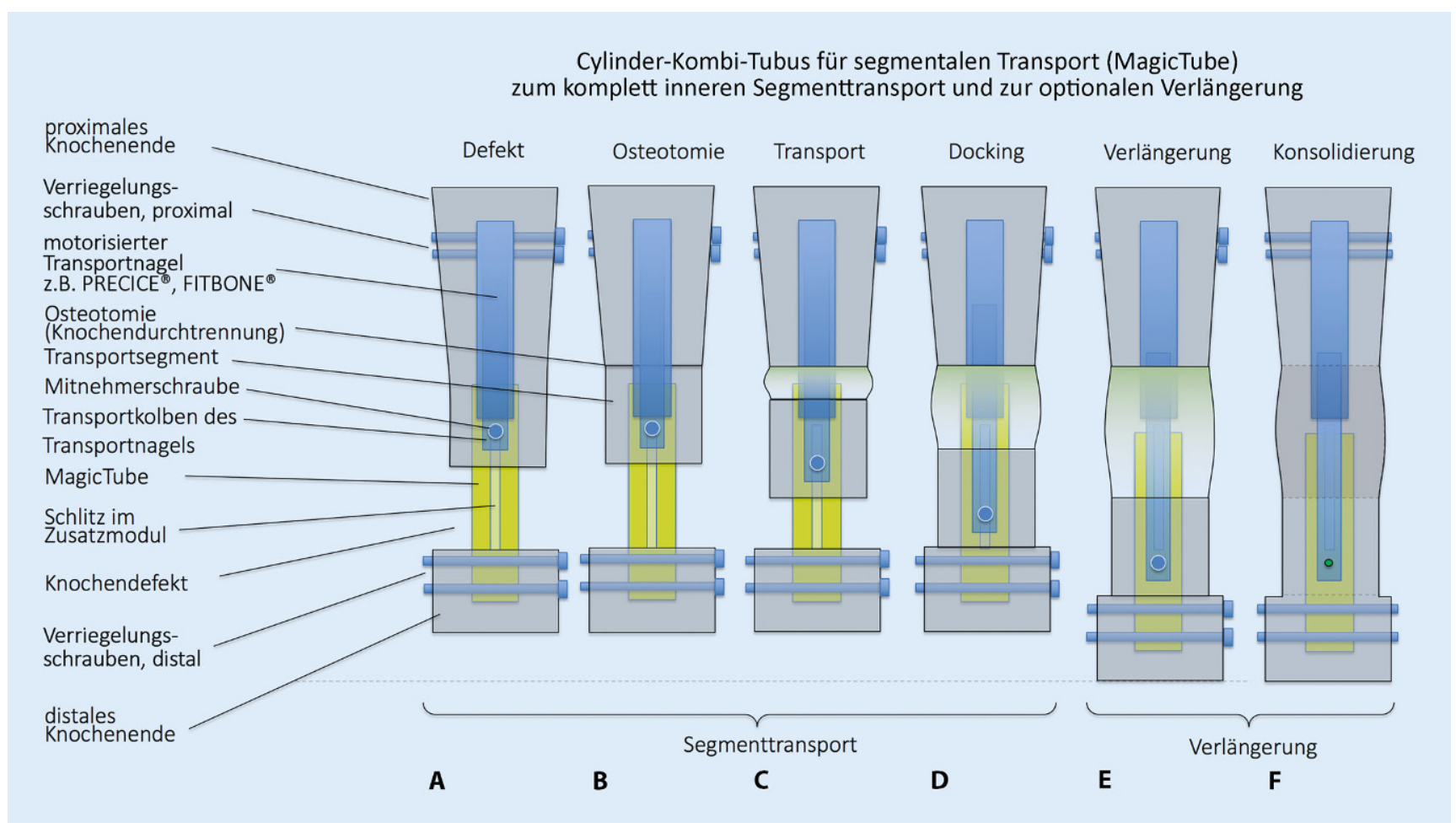

Abb. 1 ॥ Schematische Darstellung der Komponenten und wesentlichen Operationsschritte in der anterior-posterioren Ansicht; grau Knochen, blau motorisierter Knochenverlängerungsnagel, gelb MagicTube-Modul. $A$ Knochendefektsituation mit motorisiertem Verlängerungsnagel und MagicTube; $B$ Osteotomie durchgeführt, noch kein Transport; CBeginn des Knochensegmenttransports; D Docking-Situation; $E$ optionale Verlängerung: das Transportsegment schiebt das distale Hauptfragment vor sich her und führt zur zusätzlichen Verlängerung; $F$ nach Konsolidierung des Segmentdefekts ist dieser stabil gefüllt und der Knochen auf die gewünschte Länge verlängert worden. (Nach Krettek und EI Naga [5]; mit freundl. Genehmigung von Wolters Kluwer Health. Dieser Inhalt ist nicht Teil der Open-Access-Lizenz)

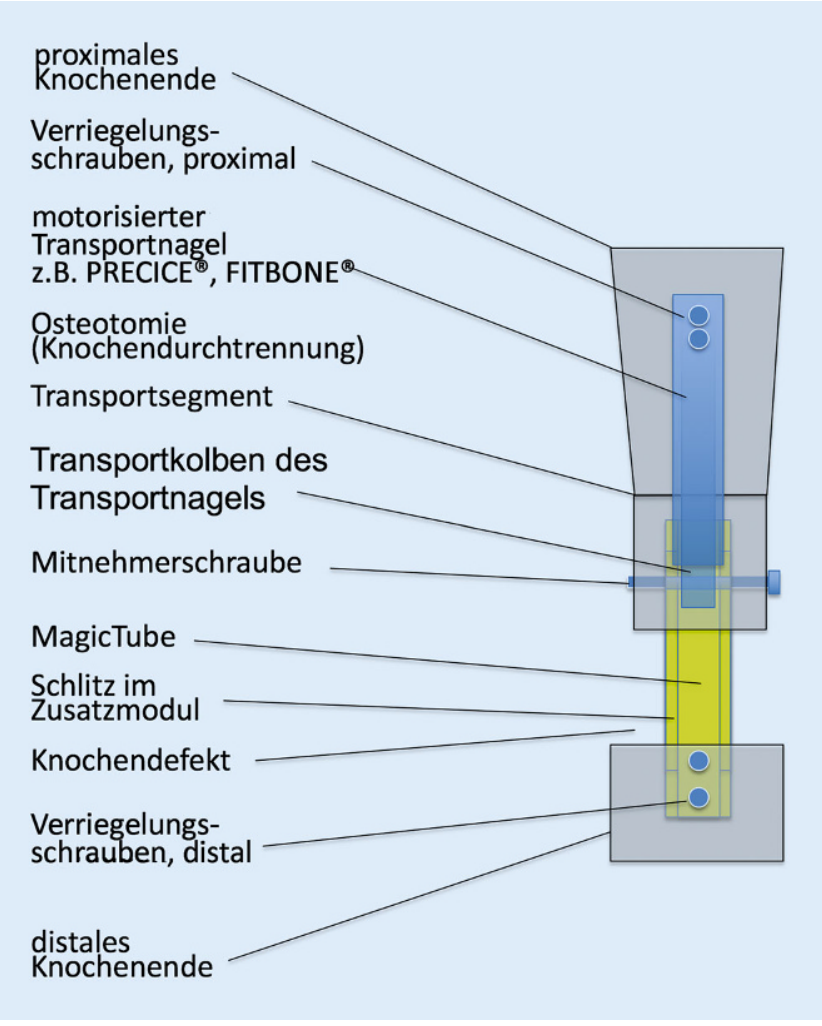

Abb. $2<$ Schematische Darstellung der Komponenten des Verfahrens in der Seitenansicht; grau Knochen, blau motorisierter Knochenverlängerungsnagel, gelb MagicTube-Modul. (Nach Krettek und El Naga [5]; mit freundl. Genehmigung von Wolters Kluwer Health. Dieser Inhalt ist nicht Teil der Open-Access-Lizenz) che Knochenverlängerung in Fällen, in denen eine Verkürzung besteht. Dies ist bis heute mit keinem anderen inneren motorisierten KST-System ohne zusätzliche operative Eingriffe möglich. Das ist deshalb von Bedeutung, weil bei dem hier zur Diskussion stehenden Patientengut fast regelmäßig auch eine Verkürzung vorliegt. Da sowohl der KST als auch die Knochenverlängerung im Körperinneren erfolgen, können Risiken, Nachteile und negative Nebenwirkungen externer Transportsysteme vermieden werden.

\section{Beschreibung des Implantat- moduls}

Das Modul besteht aus einem einfachen Zylinder mit gegenüberliegenden Schlitzen und Bohrungen an einem Ende. Das Modul kann mit jedem handelsüblichen motorisierten Knochenverlängerungsnagel kombiniert werden. Es nutzt die Energie des motorisierten Verlänge- 
rungsnagels für einen inneren KST und ggf. die Knochenverlängerung.

Das Modul wird am Nagelende aufgesteckt. Die Bohrung für die Nagelverriegelungsschraube fluchtet mit dem Doppelschlitz im Zusatzmodul. Eine Verriegelungsschraube fixiert das Transportsegment am Transportkolben des motorisierten Nagels. Das Zusatzmodul wird mit Verriegelungsschrauben z. B. am distalen Hauptfragment fixiert. Je nach Situation kann entweder ein alleiniger KST durchgeführt oder der Knochen bei fortgesetzter Aktivierung des Motorantriebs ohne weiteren Eingriff zusätzlich verlängert werden.

\section{Chirurgische Technik und Fallbeschreibung}

\section{Anwendungsbeispiele}

\section{Tibia}

Vorgeschichte. Ein 74 Jahre alter Patient erlitt eine offene distale Tibiafraktur vom Grad IIIA (AO/OTA42A) mit begleitender Fibulafraktur. Die Komplikationswunde wurde initial debridiert und die Frakturen stabilisiert (offene Reposition und innere Fixierung [ORIF] der Tibia: mediale 14-Loch-Titan-Kompressionsplatte und Zugschraube, ORIF der Fibula: laterale Titan-1/3-Rohrplatte; • Abb. 3). Etwa ein Jahr später präsentierte sich der Patient in der Medizinischen Hochschule Hannover mit Schmerzen (visuelle analoge Skala [VAS] 6-8) und der Unfähigkeit, ohne 2 Unterarmgehstützen zu gehen. In Höhe der Tibiaplatte fand sich in der Vorgeschichte eine Fistelöffnung bei dem ansonsten gesunden Patienten (Nichtraucher, kein Diabetes; - Abb. 3). Röntgenaufnahmen und CTScans zeigten eine atrophe Pseudarthrose der Tibia mit Randsklerose der tibialen Fragmentenden und verheilter Fibula. Es bestanden eine leichte $3^{\circ}$-Valgus-Deformität und ein $25^{\circ}$-Außenrotation-Fehler im Vergleich zur unverletzten Seite. Auf Höhe der Pseudarthrose zeigten sich dunkel verfärbte, indurierte Weichteile. Der Fuß hatte normale Pulse mit intakter Sensorik und motorischer Funktion. Es wurde ein leichter Spitzfuß mit einer Beweglichkeit im oberen Sprunggelenk von Dorsal-/Plantarflexion $0^{\circ} / 10^{\circ} / 30^{\circ}$

Unfallchirurg 2018 $\cdot 121: 884-892$ https://doi.org/10.1007/s00113-018-0549-9

(c) Der/die Autor(en) 2018

\section{Krettek}

MagicTube: Neue Möglichkeiten zum komplett internen Knochensegmenttransport und optionaler Verlängerung. Neues Zusatzmodul für motorisierte Verlängerungsnägel zur Behandlung großer Knochendefekte

\section{Zusammenfassung}

Der Knochensegmenttransport (KST) ist eine effektive Behandlungsmethode für segmentale Knochendefekte, aber die bisher vorhandene Notwendigkeit einer externen Fixateurkonstruktion während der Transportphase ist insbesondere am Femur ein großer Nachteil. Um eine externe Fixierung zu vermeiden, wurde vom Autor der MagicTube, ein Cylinder-Kombi-Tubus als segmentales Transport(CKTST, MagicTube)Modul, entwickelt, der sich für die Kombination mit einem der kommerziell erhältlichen motorisierten Verlängerungsnägel eignet.
Dieses MagicTube-Modul ermöglicht den komplett internen KST und bei Bedarf auch eine optionale Knochenverlängerung. Das Konzept und die chirurgische Technik von MagicTube werden anhand klinischer Fallbeispiele an Tibia und Femur beschrieben, illustriert und diskutiert.

\section{Schlüsselwörter}

Intramedulläre Frakturfixation · Knochennägel · Knochenregeneration · Knochentransport · Knochensegmenttransport

\section{MagicTube: new possibilities for completely internal bone segmental transport and optional lengthening. New additional module for motorized lengthening nails for treatment of large bone defects}

\section{Abstract}

Segmental bone transport is an effective method of treatment for segmental defects but the previous need for external fixation constructs during the transport phase is a great disadvantage, especially in the femur. In order to avoid external fixation, a new cylindrical combitube segmental transport (CKTST, MagicTube) module for combination with a commercially available motorized lengthening nail was developed by the author. This MagicTube module enables completely internal segmental bone transport and also enables optional lengthening if needed. The concept and surgical technique of MagicTube are described, illustrated, discussed and exemplified by clinical cases involving the tibia and femur.

\section{Keywords}

Fracture fixation, intramedullary · Bone nail . Bone regeneration - Bone transport - Bone segment transport festgestellt; die Kniegelenkbeweglichkeit war seitengleich normal.

Vorbereitung. Nach mehreren ausführlichen Informationsgesprächen über Vor- und Nachteile, Möglichkeiten und Risiken verschiedener Behandlungsoptionen (Entscheidungsmatrix: - Tab. 1) entschied sich der Patient für eine Segmentresektion, Lappendeckung und einen komplett internen Segmenttransport mit dem MagicTube-Modulsystem. Das MagicTube-Modul ist kein kommerziell erhältliches Implantatmodul, sondern eine Sonderanfertigung im Sinne eines Heilversuchs. Produktion,
Lagerung und Implantation entsprachen den deutschen und den europäischen medizinischen Vorschriften (Medizinproduktegesetz). Die Verantwortung liegt ganz beim Chirurgen. Die $\mathrm{Zu}$ stimmung des örtlichen Ethikkomitees wurde eingeholt.

Operationstechnik. In der ersten Operation unterzog sich der Patient einem gründlichen Weichteil- und Knochendébridement. Nach der Entfernung des nekrotischen Knochens verblieb ein Tibiadefekt von $90 \mathrm{~mm}$. Dieser Defekt wurde mit einem Antibiotika(Gentamicin)haltigen Zementabstandshalter gefüllt 

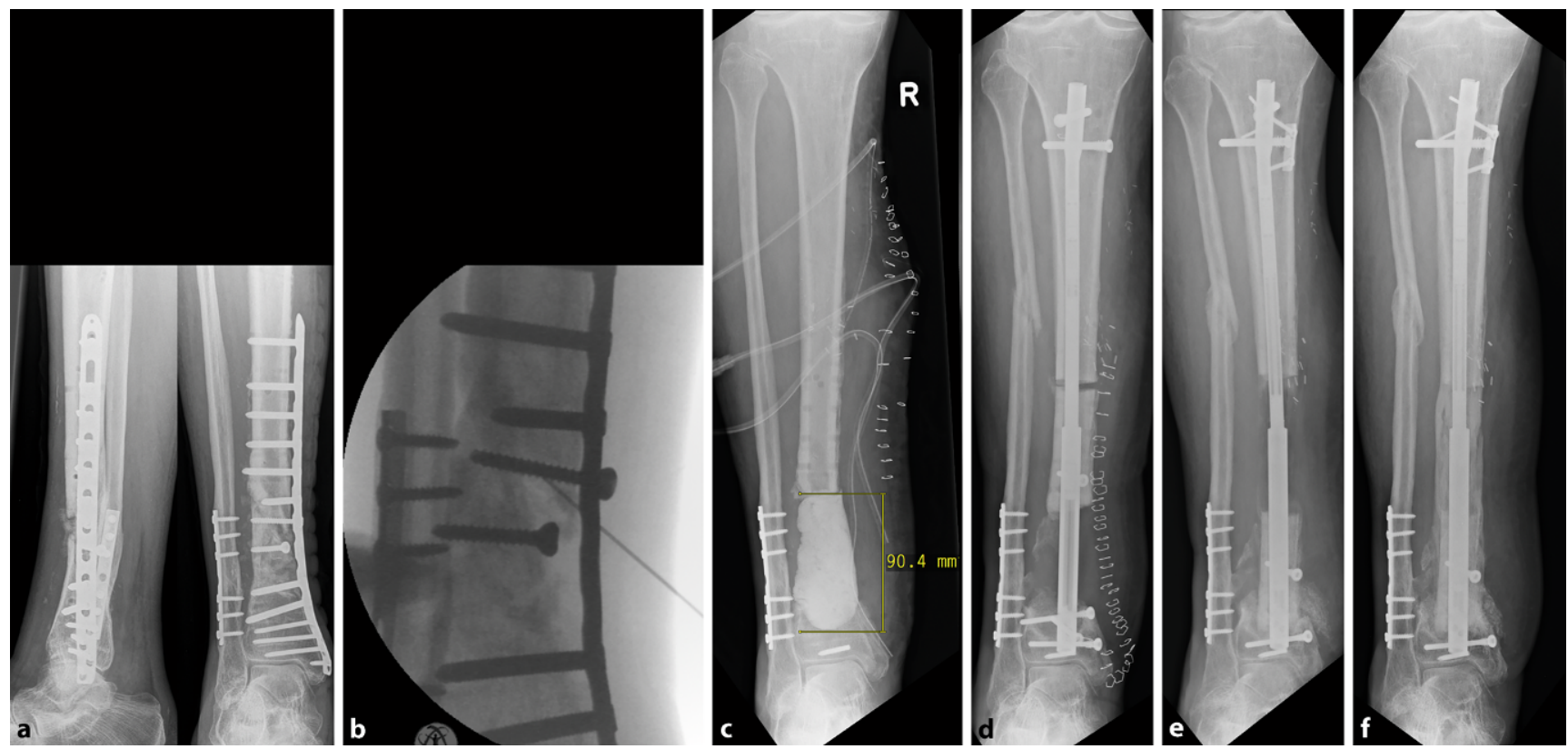

Abb. $3 \Delta$ Röntgenverlaufsserie mit den verschiedenen Stadien der Behandlung. a Vor Débridement; $\mathbf{b}$ Fisteldarstellung; c $90 \mathrm{~mm}$ Defekt nach Débridement, gefüllt mit Zementabstandhalter; $\mathbf{d}$ Entfernung des Zements. Eingesetzter motorisierter Knochenverlängerungsnagel mit aufgestecktem MagicTube am Anfang des Segmenttransports; e nach Andocken des Transportsegments und Entfernen einer interferierenden distalen Verriegelungsschraube; fam Ende des Transports knöchern konsolidierte Transportstrecke

und die Wunde mit einem Vakuumsystem verschlossen. Einige Tage später wurde der Defekt mit einem freien gefäßgestielten myokutanen Latissimus-dorsiLappen von den Kollegen aus der plastischen Chirurgie gedeckt. Die Wundheilung verlief problemlos. Der Patient wurde mit einer Unterschenkelschiene plus Fußeinschluss (Teilbelastung 15-20 kg) unter sehr milden Schmerzen (VAS 1-2) mobilisiert. Nach kompletter Ab- und Einheilung des freien Lappens wurde die Behandlung fortgeführt. Der Lappen wurde gehoben, der Zementabstandshalter entfernt und die Torsionsfehlstellung nach einer Fibulaosteotomie korrigiert. Nach einem transligamentären Zugang zur proximalen Tibia wurde ein motorisierter Knochenverlängerungsnagel (PRECICE ${ }^{\circledR}$, Fa. Nuvasive, San Diego, Kalifornien) mit dem aufgesteckten MagicTube-Modul antegrad in den Tibiamarkraum eingeführt und zentral in der sehr kurzen distalen Tibia platziert. Das Ende des MagicTube-Moduls kam direkt auf einer verbliebenen abgebrochenen Plattenschraube zu liegen, die beim Débridement belassen worden war. Der motorisierte Nagel wurde proximal in der proximalen Tibia ver- riegelt ( $\bullet$ Abb. 4 und 5). Das MagicTubeModul wurde im distalen Tibiafragment mithilfe von 2 Verriegelungsschrauben verriegelt, nachdem sichergestellt war, dass 1) der Schlitz des MagicTube-Moduls sich in einer Ebene mit den distalen Verriegelungslöchern des motorisierten Nagels befand und 2) die tibiale Torsion spiegelsymmetrisch zur kontralateralen Seite ausgerichtet war.

Dann wurde die Mitnehmerschraube im Knochentransportsegment gesetzt, und zwar 1) durch den Knochen des Transportsegments, 2) durch die miteinander fluchtenden Öffnungen im MagicTube-Modul und 3) durch den Transportkolben des Transportnagels.

Anschließend wurde das Transportsegment mithilfe einer Gigli-Säge vom proximalen Hauptfragment getrennt. Die Transportfunktion wurde mit dem Magnetantrieb getestet.

\section{Postoperativer Behandlungsverlauf.}

Die Wundheilung war unproblematisch. Der Patient konnte mit einer Unterschenkelschiene plus Fußeinschluss (Teilbelastung 15-20 kg) sehr schmerzarm (VAS 1-2) mobilisiert werden.
Aufgrund seines hohen Alters des Patienten (74 Jahre) wurde der Transport erst 3 Wochen nach der Osteotomie gestartet. Im Hinblick auf die altersbedingt eingeschränkte Regenerationsfähigkeit wurde die Transportrate mit $0,25 \mathrm{~mm} /$ Tag zunächst sehr niedrig gehalten.

\section{》) Ist die Defektstrecke größer als der Kolbenhub, muss ein Pit-stop-Manöver durchgeführt werden}

Wöchentlich wurden Röntgenaufnahmen und Ultraschallkontrollen durchgeführt. Nach 4 Wochen zeigte sich eine lediglich sehr geringe Dichte im Regenerat, sodass der Transport für weitere 2 Wochen in die entgegengesetzte Richtung gesteuert wurde (Kompression/Ziehharmonikatechnik), um das Regenerat besser reifen zu lassen. Drei Monate nach der Osteotomie wurde die Transportrate auf $1 \mathrm{~mm} / \mathrm{Tag}$ erhöht, und in den Röntgenaufnahmen wurde die beginnende homogene Verkalkung des Transportkallus ersichtlich. 


\section{Leitthema}

Tab. 1 Entscheidungsmatrix für große segmentale Knochendefekte an Tibia und Femur [5]

\begin{tabular}{|c|c|c|c|c|c|c|c|}
\hline Methode & Transport & Fixation & $\begin{array}{l}\text { Ohne grö- } \\
\text { ßere Kno- } \\
\text { chentrans- } \\
\text { plantation }\end{array}$ & $\begin{array}{l}\text { Wieder- } \\
\text { herstel- } \\
\text { lung des } \\
\text { Knochen- } \\
\text { rohrs }\end{array}$ & $\begin{array}{l}\text { Möglichkeiten } \\
\text { zur Knochen- } \\
\text { verlängerung } \\
\text { ohne } \\
\text { weiteren } \\
\text { Eingriff }\end{array}$ & $\begin{array}{l}\text { Hohes Risiko } \\
\text { der Knie- } \\
\text { gelenkkon- } \\
\text { traktur bei } \\
\text { Anwendung } \\
\text { am Femur }\end{array}$ & Bemerkungen \\
\hline Ilizarov $[3,12]$ & $\begin{array}{l}\text { Externer } \\
\text { Transport } \\
\text { 1/3 Zeit }\end{array}$ & $\begin{array}{l}\text { Externe } \\
\text { Fixation } \\
2 / 3 \text { Zeit }\end{array}$ & $\mathrm{Ja}$ & $\mathrm{Ja}$ & $\mathrm{Ja}$ & Ja & $\begin{array}{l}\text { Sehr lange Präsenz des Fixa- } \\
\text { teur externe (z. B. } 90 \text { mm De- } \\
\text { fekt: Transportzeit } 3 \text { Mona- } \\
\text { te + Konsolidierungszeit } 6 \text { Mo- } \\
\text { nate = insgesamt } 9 \text { Monate) } \\
\text { Schwierigkeiten der Fixierung des } \\
\text { Fixateurs in sehr kurzen Hauptfrag- } \\
\text { menten }\end{array}$ \\
\hline $\begin{array}{l}\text { Monorail }[8,11 \text {, } \\
14]\end{array}$ & $\begin{array}{l}\text { Externer } \\
\text { Transport } \\
\text { 1/3 Zeit }\end{array}$ & $\begin{array}{l}\text { Intern } \\
\text { (Nagel, } \\
\text { Platte) }\end{array}$ & $\mathrm{Ja}$ & $\mathrm{Ja}$ & $\begin{array}{l}\text { Ja (modifizierte } \\
\text { Konstruktion) }\end{array}$ & $\mathrm{Ja}$ & $\begin{array}{l}\text { Lange Präsenz des Fixateur externe } \\
\text { (z. B. } 90 \text { mm Defekt: Transportzeit } \\
3 \text { Monate) } \\
\text { Schwierigkeiten der Fixierung des } \\
\text { Fixateurs in sehr kurzen Hauptfrag- } \\
\text { menten }\end{array}$ \\
\hline \multirow[t]{2}{*}{ Masquelet $[9,10]$} & \multirow[t]{2}{*}{ Intern } & $\begin{array}{l}\text { Intern } \\
\text { (Platte) }\end{array}$ & Nein & $\begin{array}{l}\text { in der } \\
\text { Regel } \\
\text { Nein }\end{array}$ & Nein & Nein & $\begin{array}{l}\text { Sehr großes Volumen an benötig- } \\
\text { tem Knochenttransplantat } \\
\text { Entnahmemorbidität } \\
\text { Rigider Knochenblock statt Kno- } \\
\text { chenrohr, erhöhtes Risiko für Ermü- } \\
\text { dungsfrakturen }\end{array}$ \\
\hline & & $\begin{array}{l}\text { Intern } \\
\text { (Nagel) }\end{array}$ & Nein & $\mathrm{Ja}$ & Nein & Nein & $\begin{array}{l}\text { Sehr großes Volumen an benötig- } \\
\text { tem Knochenttransplantat } \\
\text { Entnahmemorbidität }\end{array}$ \\
\hline MagicTube [5] & Intern & $\begin{array}{l}\text { Intern } \\
\text { (Nagel) }\end{array}$ & $\mathrm{Ja}$ & $\mathrm{Ja}$ & $\mathrm{Ja}$ & Nein & $\begin{array}{l}\text { Neues Konzept, mehr Daten benö- } \\
\text { tigt }\end{array}$ \\
\hline
\end{tabular}

\section{Tab. 2 Grundsätzliche operative Teilschritte beim Pit-stop-Manöver}

\section{Teilschritte}

1. Temporäre mechanische Sicherung des Transportsegments

2. Lösen der Transportmitnehmerfixierung

3. Rückführen des Transportkolbens in die Ausgangssituation

4. Erneute Verriegelung des Transportsegments

5. Entfernen der temporären mechanischen Sicherung des Transportsegments

6. Wiederaufnahme des Knochensegmenttransports

\section{Bemerkung}

Z.B. über temporären Fixateur externe oder temporäre (winkelstabiler) Platte

In der Regel Mitnehmerverriegelungsschraube, bei ungünstiger geometrischer Situation auch Mitnehmerplatte oder Drahtseil

In normaler Transportgeschwindigkeit würde dies $7 \mathrm{~min} / \mathrm{mm}$ dauern, mithilfe des "fast distractor" (Fa. NuVasive, Inc., San Diego, CA, USA) erfolgt dieser Prozess um ein Vielfaches schneller, vorausgesetzt das Implantat ist temporär ausgebaut.

Mitnehmerschraube. Wenn das Verriegelungsloch des Transportkolbens außerhalb des Transportsegments liegt, muss eine Hilfskonstruktion zur Segmentmitnahme etabliert werden (z. B. Mitnehmerplatte oder Drahtseil)
Um ein hindernisfreies Andocken zu ermöglichen, musste in der Endphase die proximale Schraube der distalen Verriegelungsschraubengruppe entfernt werden (• Abb. 3).

Pit-stop-Manöver. Der Kolbenhub von Verlängerungsnägeln ist begrenzt und beträgt meist zwischen 50 und $80 \mathrm{~mm}$. Ist die Defektstrecke größer als der Kolbenhub, muss ein Pit-stop-Manöver durchgeführt werden. Darunter werden der temporäre Transportstopp, die temporäre mechanische Sicherung des Transportsegments, das Lösen der Transportfixierung (Verriegelungsschraube), das Rückführen des Transportkolbens in die Ausgangssituation und das Entfernen der temporären mechanischen Sicherung des Transportsegments verstanden. Anschließend kann der unterbrochene Transport wieder aufgenommen werden (•Tab. 2). 


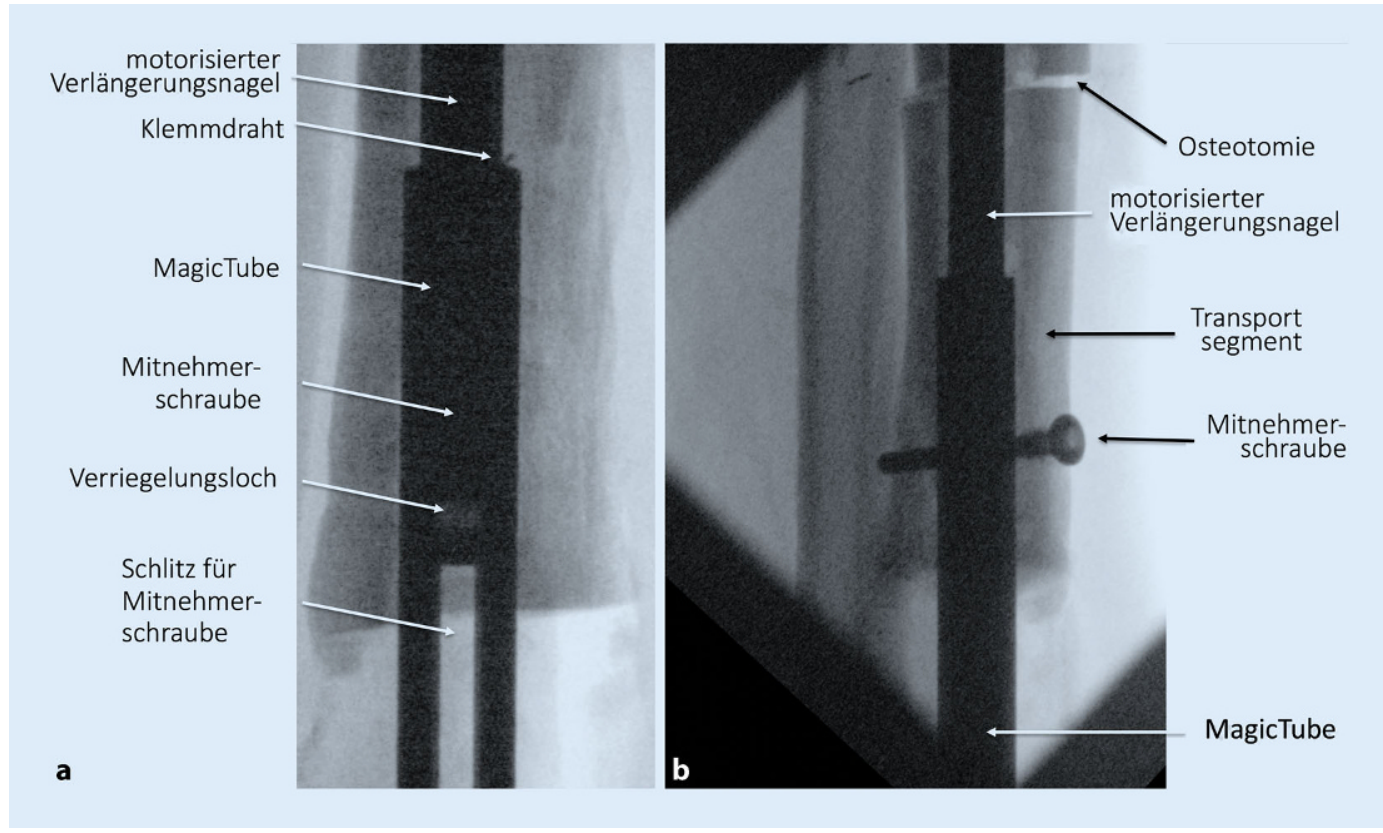

Abb. $4<$ Durchleuchtungsbilder nach Einbringen und Verriegelung des MagicTube-Moduls mit dem Transportsegment. Transportsegment vor (a) und nach der Osteotomie (b). Die Verriegelungsschraube wird durch den Knochen, die Schlitze des MagicTube und durch den Transportkolben des Nagels platziert. (Nach Krettek und El Naga [5]; mit freundl. Genehmigung von Wolters Kluwer Health. Dieser Inhalt ist nicht Teil der Open-Access-Lizenz)

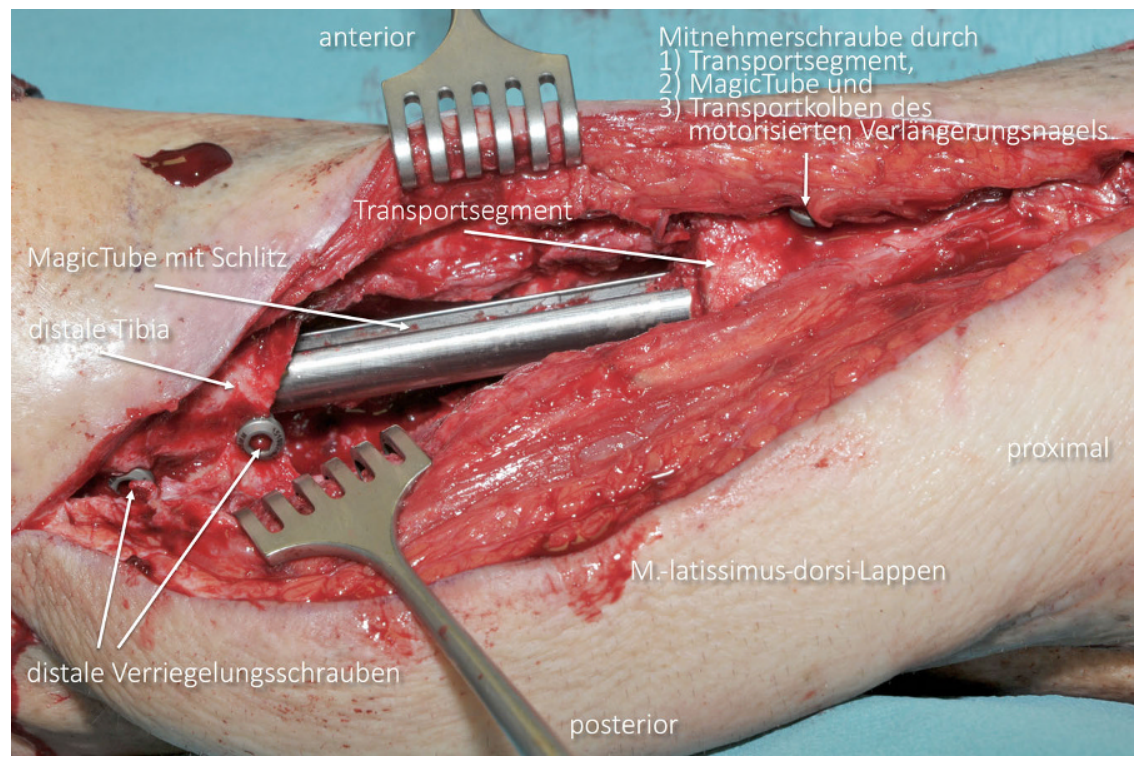

Abb. 5 ॥ Intraoperatives Foto. MagicTube-Modul im Defekt sichtbar, mit durch das Transportsegment und im distalen Hauptfragment platzierten Verriegelungsschrauben. (Nach Krettekund El Naga [5]; mit freundl. Genehmigung von Wolters Kluwer Health. Dieser Inhalt ist nicht Teil der Open-Access-Lizenz)

Komplikationen. Während des Transports waren Schraubenlockerungsphänomene im Bereich der proximalen Verriegelungsschrauben im Nagel zu verzeichnen. Diese Probleme wurden mit dem Aufbringen kleiner Plättchen adressiert.

\section{Femur}

Ein 39-jähriger Patient stellte sich nach einer auswärts mithilfe einer Plattenosteosynthese behandelten Schussverlet- zung mit femoralem Knochenverlust, Pseudarthrose und Plattenbruch vor. Nach weiteren debridierenden und stabilisierenden Voroperationen betrug der Gesamtknochenverlust bei einer Kombination aus $94 \mathrm{~mm}$ Segmentdefekt und $28 \mathrm{~mm}$ Verkürzung insgesamt $122 \mathrm{~mm}$ (• Abb.6). Zunächst erfolgte ein weiteres gründliches Weichteil- und Knochendébridement. Der Knochen wurde mit einem intraoperativ gefertigten intramedullären Nagel, bestehend aus einem
Bündelnagel mit Knochenzementmantel, geschient. Ein verbleibender Knochensegmentdefekt wurde mit antibiotikahaltigem Knochenzement als Platzhalter gefüllt. Nachdem mehrfache Punktionsaspirationen keinen mikrobiologischen Keimnachweis ergaben, wurde in einem weiteren Eingriff ein motorisierter Verlängerungsnagel mit aufgestecktem MagicTube-Modul antegrad eingebracht und der Transportnagel proximal, der MagicTube distal verriegelt. $\mathrm{Da}$ die Spitze des Transportkolbens über das Transportfragment hinausragte, wurde das distale Ende des Transportkolbens mithilfe einer $3,5 \mathrm{~mm}$ winkelstabilen Platte als Mitnehmervorrichtung mit dem osteotomierten Transportsegment winkelstabil verbunden. Aufgrund des begrenzten Transporthubs des Transportnagels waren Pit-stop-Manöver erforderlich. Beim ersten dieser Eingriffe wurde die Mitnehmerplatte gegen einen Seilzugmechanismus ausgetauscht. Im weiteren Verlauf kam es zu einem Ermüdungsbruch des Transportseils. Der Eingriff zur Behebung dieser Komplikation wurde für ein weiteres Pit-stopManöver genutzt, um den ausgefahrenen Transportkolben wieder zurückzuholen. Der KST war nun so weit fortgeschritten, dass das Transportsegment an der Spitze des Transportkolbens über eine transossäre Mitnehmerschraube durch 


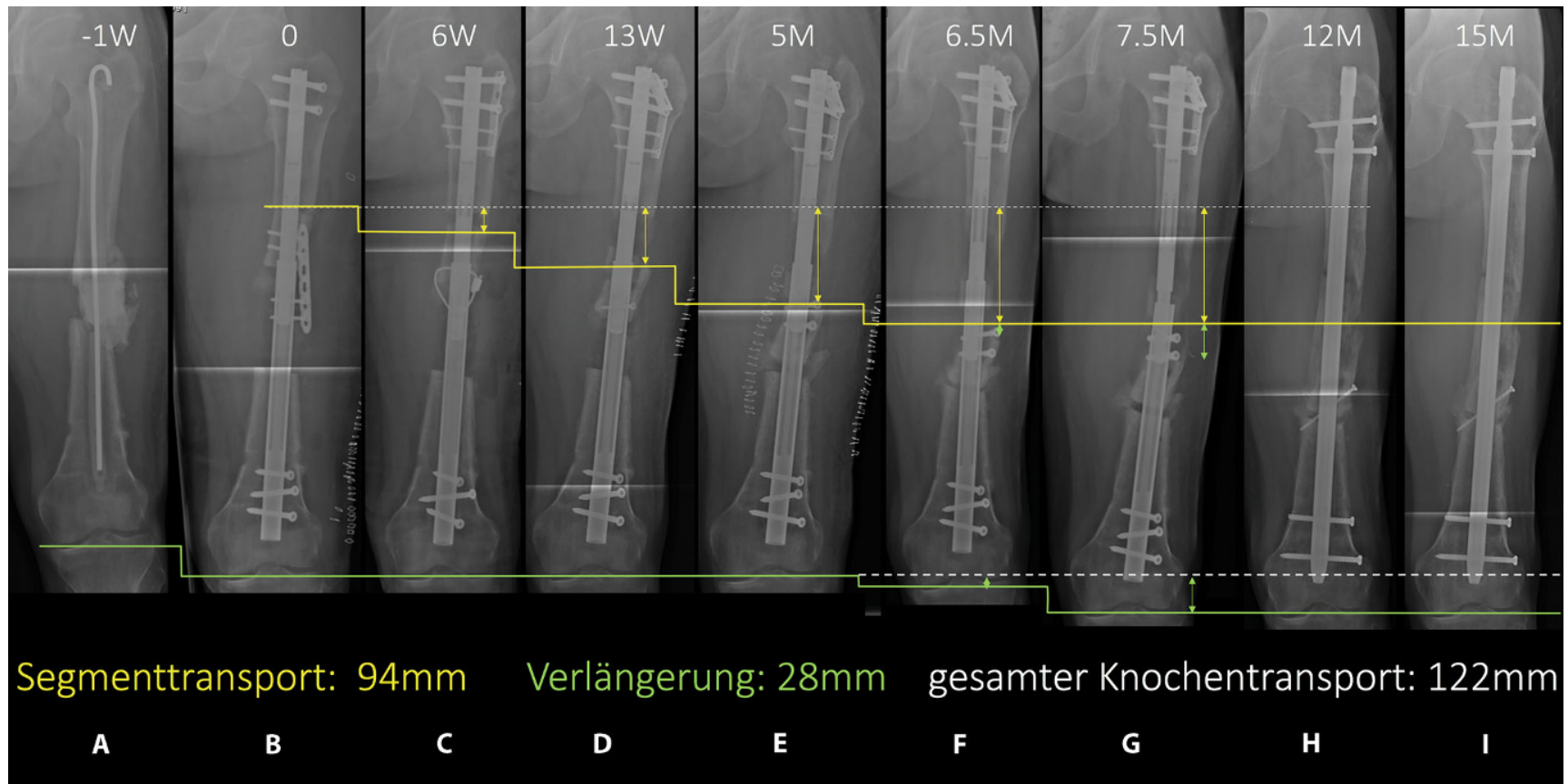

Abb. 6 \ Röntgenaufnahmen eines 39-jährigen Patienten nach Schussverletzungen mit einem langstreckigen femoralen Knochenverlust und Pseudarthrose. Kombinierter Segmenttransport und Verlängerung mit dem MagicTube-Modul. $A$ Antibiotikazement als Abstandshalter; $B$ nach Knochendébridement Einbringen eines motorisierten Verlängerungsnagels mit aufgestecktem MagicTube-Modul. Mehrfache Pit-stop-Operationen, da der maximale Transporthub des motorisierten Verlängerungsnagels begrenzt ist. Unterschiedliche Mitnehmermechanismen: 1. Mitnehmermechanismus: Platte. C 2. Mitnehmermechanismus:Drahtseil.E3. Mitnehmermechanismus:Verriegelungsschraube. F,GMit Auftreffen des Transportsegments auf das distale Fragment wird dieses vom Transportsegment weitergeschoben. Damit wird das Femur verlängert. G Der gesamte Knochentransport beträgt $122 \mathrm{~mm}$. Dieser Betrag setzt sich aus $94 \mathrm{~mm}$ Segmenttransport und $28 \mathrm{~mm}$ Verlängerung zusammen. $H$ Nach einem Jahrund Erreichen der Ziellänge wird am Ende des Transports die "docking site" mit einer Zugschraube gesichert und der Nagel gegen einen konventionellen Nagel (CFN; Fa. DePuy Synthes, West Chester, PA, USA) ausgetauscht. I Zunehmende Konsolidierung im Bereich der Docking site. - 1 W 1 Woche vor Implantation des MagicTubes, OW Unmittelbar nach Implantation des MagicTubes, 6 W 6 Wochen vor Implantation des MagicTubes

den Schlitz des MagicTube und das Verriegelungsloch des Transportkolbens fixiert werden konnte.

Mit Auftreffen des Transportsegments auf das distale Fragment wurde die Verlängerungsphase eingeleitet, indem das distale Hauptfragment vom Transportsegment vor sich hergeschoben wird. Damit wurde das verkürzte Femur verlängert. Der gesamte Knochentransport betrug also $122 \mathrm{~mm}$. Dieser Betrag setzte sich aus $94 \mathrm{~mm}$ Segmenttransport und $28 \mathrm{~mm}$ zusammen. Am Ende des Transports wurde auf dem Extensionstisch (Sicherung der Länge) in Seitenlage des Patienten ein Docking-Eingriff durchgeführt. Zunächst wurde die Docking site mit einer Zugschraube gesichert. Anschließend wurden der Verlängerungsnagel und MagicTube entriegelt, entfernt und gegen einen konventionellen Nagel (CFN; Fa. DePuy Synthes, West Chester, PA, USA) ausgetauscht (• Abb. 7).
Wegen einer Fehlbestimmung der Ausgangslänge kam es zu einer passageren und schmerzhaften Überdistraktion, die am Folgetag operativ korrigiert werden musste.

\section{Diskussion}

Das 2017 erstmals beschriebene MagicTube-Modul [5] ist das erste und bislang einzige Implantat, das im Zusammenwirken mit einem motorisierten Verlängerungsnagel einen inneren Segmenttransport und die anschließende optionale Verlängerung ohne einen weiteren operativen Eingriff zulässt. Das einfache universelle partiell geschlitzte zylindrische Transportrohr kann mit jedem motorisierten Verlängerungsnagel mit freiem Kolben kombiniert werden.

Gegenüber herkömmlichen Techniken gibt es mehrere Vorteile. Die Behandlung von großen segmentalen Knochen- defekten hat sich mit der Einführung des „Induced-membrane“-Konzepts [9, 10] deutlich verbessert. Aber die Knochenentnahmemorbidität und die Schaffung eines starren massiven Knochenblocks anstelle eines Rohres hat bei diesem Verfahren häufig zu Problemen wie z.B. Refrakturen geführt. Diese Nachteile können mit dem hier vorgestellen Verfahren vermieden werden.

Der Knochensegmenttransport (KST) kann entweder mit einem kompletten externen Befestigungs- und Transportsystem $[3,12]$ oder als externer segmentaler Transport entlang eines überbrückenden Implantats (z. B. Nagel $[8,14]$ oder Platte [11]) durchgeführt werden.

Externe Fixierung und Transport können bei schwierigen Weichteilen manchmal das einzig mögliche Verfahren sein. Häufig ist aber die äußere Fixierung mit einer Vielzahl von Schwierigkeiten, Problemen und Komplikationen verbunden 


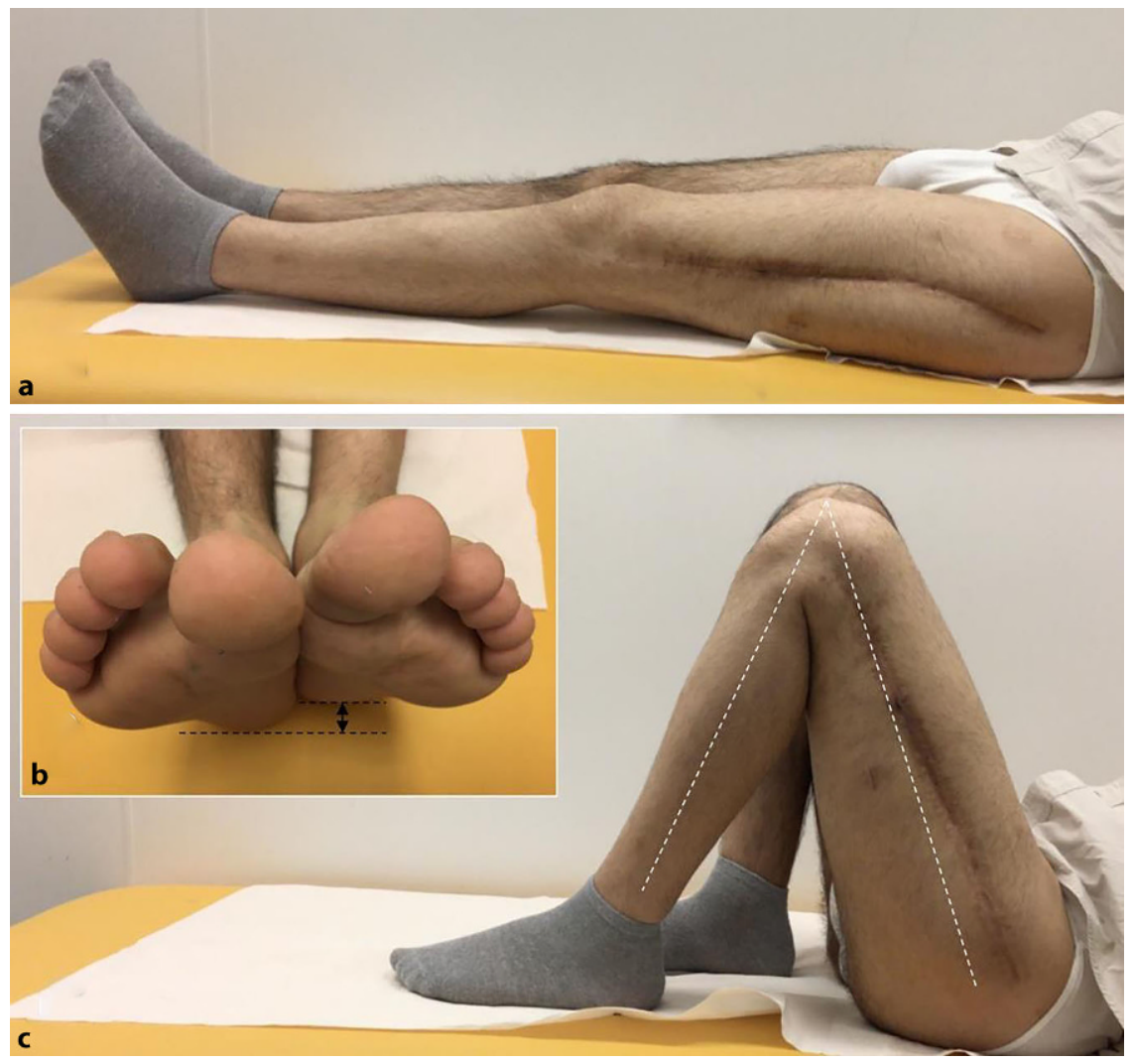

Abb. 7 A Klinische Bilder des Patienten aus - Abb. 6. a Volle Streckung; b 12 mm vor dem Transportende (gesamter Transport $122 \mathrm{~mm}$ ); c immer noch hervorragende "range of motion" (ROM) am linken Knie mit $0 / 0 / 137^{\circ}$, im Vergleich zu Gegenseite $\left(5 / 0 / 150^{\circ}\right)$ nur wenig eingeschränkt

[12]. Meist sind die weichteilperforierenden Implantate (Schanz-Schrauben, Drähte) lediglich bakteriell kontaminiert, grundsätzlich besteht aber das Risiko einer an den Implantaten fortgeleiteten Infektion in das Knocheninnere. Ein weiterer Nachteil von partiellen oder vollständigen externen segmentalen Transportkonzepten ist die Notwendigkeit der Platzierung von Schanz-Schrauben oder Drähten in den proximalen- und/oder distalen Hauptfragmenten. Das kann in kurzen Segmenten schwierig oder unmöglich sein, wie beim vorgestellten $\mathrm{Pa}$ tienten. Insbesondere am Femur kann es durch Traktopexie (Tractus iliotibialis) und Myopexie (M. quadriceps) zu Bewegungsschmerzen und u. U. sehr ausgeprägten Kniegelenkeinsteifungen und Kontrakturen kommen.

Aus diesen Gründen ziehen wir es vor, eine externe Fixierung zu vermeiden, wann immer es möglich ist. Neu entwickelte magnetisch aktivierte Nägel $[4,13,15]$ haben Vorteile gegenüber durch Gang aktivierten Systemen [2], entsprechend größer und die übertragene Energie im Vergleich zu normalgewichtigen Patienten mit schlanken Gliedmaßen geringer.

Das MagicTube-Modul hat den Vorteil, dass es eine gute Kontrolle des Transports sowie der Transportgeschwindigkeit und -richtung ermöglicht. Im vorgestellten Tibiafall war dies hilfreich und wichtig, da die Knochenregeneration des 74-jährigen Patienten weniger zuverlässig war als bei einem jüngeren Patienten. Als die Regeneration in einer routinemäßigen Ultraschalluntersuchung weniger Reflexion und Dichte zeigte als erwartet, wurde die Richtung des KST temporär 2 Wochen lang umgedreht. Ein weiterer wichtiger Vorteil ist die Tatsache, dass das MagicTube-Modul eine zusätzliche optionale Knochenverlängerung ermöglicht, da die meisten Patienten mit segmentalen Defekten auch eine mehr oder weniger große Verkürzung aufweisen.

》) MagicTube-Modul ermöglicht gute Kontrolle des Transports, seiner Geschwindigkeit und Richtung

die schwer zu kontrollieren sind (Geschwindigkeit, Stopp) und die Richtung nicht umkehren können. Magnetisch aktivierte motorisierte Nägel überwinden diese Nachteile, da sie eine gute Kontrolle von Start und Stopp sowie von Richtung und Geschwindigkeit ermöglichen. Diese werden im Einsatz mit dem MagicTube-Modul bevorzugt.

Mögliche Nachteile hängen damit zusammen, dass jedes motorisierte Nagelsystem eine gewisse Mindestlänge des Nagels benötigt, die in kurzen Fragmenten bei metaphysennahen Defekten problematisch sein kann. Unter diesen Umständen kann eine direkte Verriegelung des Transportfragments nicht möglich sein, und andere Mitnehmermethoden (Draht, Platte) müssen erwogen werden. Ein weiterer Nachteil von magnetisch motorisierten Nägeln ist die Tatsache, dass die Induktion von Energie vom Abstand zwischen dem äußeren Aktor und dem internen Empfänger im Nagel beeinflusst wird. Bei adipösen Patienten mit großem Weichteilmantel ist der Abstand
Im Rahmen des knöchernen Débridements beim Docking kommt es häufig zu einer weiteren Verkürzung. Aus diesem Grund strebt der Autor immer eine leichte Verlängerung an, wenn ein DockingEingriff mit Anfrischen der Knochenenden absehbar ist. Ein weiterer Vorteil ist, dass selbst sehr kurze Hauptfragmente mit dieser Technik adressiert werden können (• Abb. 3, 4 und 5). Hier wäre die Platzierung von Schanz-Schrauben schwierig oder unmöglich.

Einschränkungen in der Beurteilung der Methode bestehen durch die bislang geringe Patientenzahl. Weitere Untersuchungen sind erforderlich.

\section{Fazit für die Praxis}

- Das MagicTube-Modul ist einfach, ohne bewegliche Teile und kostengünstig herstellbar.

- Es handelt sich um ein universelles modulares Konzept; der MagicTube 
ist mit jedem handelsüblichen Knochentransportnagel kombinierbar.

- Bei Einsatz im Femur minimiert das Modul als inneres Verfahren das Risiko für Kniegelenkkontrakturen und -einsteifungen.

- Das MagicTube-Modul erlaubt als weltweit einziges internes Verlängerungsimplantat den Übergang vom Segmenttransport in die optionale Beinverlängerung ohne zusätzlichen operativen Eingriff.

\section{Korrespondenzadresse}

Prof. Dr. Christian Krettek, FRACS, FRCSEd Medizinische Hochschule Hannover (MHH) Carl-Neuberg-Str. 1, 30625 Hannover, Deutschland

Krettek.Christian@mh-hannover.de

Danksagung. Dank gilt den Mitarbeitern des Instituts für Diagnostische und Interventionelle Radiologie (Bildgebung) und den Mitarbeitern der Klinik für Plastische, Ästhetische, Hand- \& Wiederherstellungschirurgie (Lappendeckung) der Medizinischen Hochschule Hannover. Besonderer Dank gilt der TraumaStiftung gGmbH und ihrer langjährigen Förderin Frau Madeleine Winter-Schulze, ohne deren stetige Unterstützung diese Entwicklung nicht möglich gewesen wären.

\section{Einhaltung ethischer Richtlinien}

Interessenkonflikt. C. Krettek gibt an, dass kein Interessenkonflikt besteht.

Soweit der Beitrag personenbezogene Daten enthält, wurde von den Patienten eine zusätzliche Einwilligung nach erfolgter Aufklärung eingeholt. Dieser Beitrag beinhaltet keine Studien an Menschen oder Tieren.

Open Access. Dieser Artikel wird unter der Creative Commons Namensnennung 4.0 International Lizenz (http://creativecommons.org/licenses/by/4.0/deed. de) veröffentlicht, welche die Nutzung, Vervielfältigung, Bearbeitung, Verbreitung und Wiedergabe in jeglichem Medium und Format erlaubt, sofern Sie den/die ursprünglichen Autor(en) und die Quelle ordnungsgemäßnennen, einen Linkzur Creative Commons Lizenz beifügen und angeben, ob Änderungen vorgenommen wurden.

\section{Literatur}

1. Baumgart R, Betz A, Schweiberer L (1997) A fully implantable motorized intramedullary nail for limb lengthening and bone transport. Clin Orthop Relat Res 343:135-143

2. Cole JD, Justin D, Kasparis T, DeVlught D, Knobloch C (2001) The intramedullary skeletal kinetic distractor (ISKD): first clinical results of a new intramedullary nail for lengthening of the femur and tibia. Injury 32(Suppl4):SD129-SD139

3. Feibel RJ, Uhthoff HK (2005) Primary llizarov ankle fusion for nonreconstructable tibial plafond fractures. Oper Orthop Traumatol 17(4/5):457-480

4. Kirane YM, Fragomen AT, Rozbruch SR (2014) Precision of the PRECICE internal bone lengthening nail. Clin Orthop Relat Res 472(12):3869-3878

5. Krettek C, El Naga A (2017) All internal segmental bone transport and optional lengthening with a newly developed universal cylinder-kombitube module for motorized nails-description of a surgical technique. J Orthop Trauma 31(Suppl 5):S39-S41

6. Laubscher M, Mitchell C, Timms A, Goodier D, Calder P (2016) Outcomes following femoral lengthening: an initial comparison of the precice intramedullary lengthening nail and the LRS external fixator monorail system. Bone Joint J 98$\mathrm{B}(10)$ :1382-1388

7. Liodakis E, Kenawey M, Krettek C, Ettinger M, Jagodzinski M, Hankemeier S (2011) Segmental transports for posttraumatic lower extremity bone defects: are femoral bone transports safer than tibial? Arch Orthop Trauma Surg 131(2):229-234

8. Liodakis E, Kenawey M, Krettek C, Wiebking U, Hankemeier S (2011) Comparison of 39 posttraumatic tibia bone transports performed with and without the use of an intramedullary rod: the long-term outcomes. Int Orthop 35(9):1397-1402

9. Masquelet AC, Fitoussi F, Begue T, Muller GP (2000) Reconstruction of the long bones by the induced membrane and spongy autograft. Ann Chir Plast Esthet 45(3):346-353

10. Mauffrey C, Hake ME, Chadayammuri V, Masquelet AC (2016) Reconstruction of long bone infections using the induced membrane technique: tips and tricks. J Orthop Trauma 30(6):e188-e193

11. Oh CW, Apivatthakakul T, Oh JK, Kim JW, Lee HJ, Kyung HS et al (2013) Bone transport with an external fixator and a locking plate for segmental tibial defects. Bone Joint J95-B(12):1667-1672

12. Paley D (1990) Problems, obstacles, and complications of limblengthening by the llizarov technique. Clin Orthop Relat Res 250:81-104

13. Paley D (2015) PRECICE intramedullary limb lengthening system. Expert Rev Med Devices 12(3):231-249

14. Raschke MJ, Mann JW, Oedekoven G, Claudi BF (1992) Segmental transport after unreamed intramedullary nailing. Preliminary report of a "Monorail" system. Clin Orthop Relat Res 282:233-240

15. Schiedel FM, Vogt B, Tretow HL, Schuhknecht B, Gosheger G, Horter MJ et al (2014) How precise is the PRECICE compared to the ISKD in intramedullary limb lengthening? Reliability and safety in 26 procedures. Acta Orthop 85(3):293-298
Bildgebende Diagnostik des Fußes

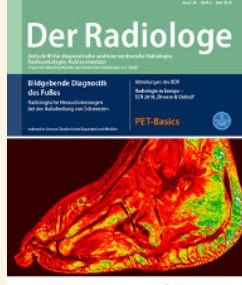

„Der menschliche Fuß ist ein Kunstwerk aus $26 \mathrm{Kno}$ chen, 107 Bändern und 19Muskeln." Dieses Zitat von Leonardo da Vinci zeigt, dass es lohnenswert ist, sich mit den wesentlichen Aspekten der Anatomie, Pathologie und den Bildgebungsbefunden, die dem Radiologen bei der diagnostischen Aufarbeitung von Schmerzen am Fuß begegnen, zu beschäftigen.

Informieren Sie sich in der Ausgabe 05/2018 von Der Radiologe unter anderem über

- Erwartungen des Orthopäden vom Radiologen bei der bildgebenden Diagnostik

- Typische Frakturen - welche sollte der Radiologe kennen?

- Knorpelbildgebung, Entzündungen und Fehlbildungen

- Tumoren und tumorähnliche Läsionen im Fuß

Suchen Sie noch mehr zum Thema? Mit e.Med - den maßgeschneiderten Fortbildungsabos von Springer Medizin - haben Sie Zugriff auf alle Inhalte von SpringerMedizin.de. Sie können schnell und komfortabel in den für Sie relevanten Zeitschriften recherchieren und auf alle Inhalte im Volltext zugreifen.

Weitere Infos zu e.Med finden Sie auf springermedizin.de unter "Abos" 